\title{
lleocolic intussusception in pediatric SARS-CoV-2 patients: experience at a tertiary pediatric center
}

\author{
Rida Salman ${ }^{1} \cdot$ Andrew C. Sher ${ }^{1} \cdot$ Marla B. K. Sammer $^{1}$ J. Ruben Rodriguez ${ }^{2} \cdot$ Sohail R. Shah $^{2} \cdot$ Victor J. Seghers $^{1}$ (i)
}

Accepted: 2 January 2022 / Published online: 8 January 2022

(c) The Author(s), under exclusive licence to Springer-Verlag GmbH Germany, part of Springer Nature 2022

\begin{abstract}
Purpose COVID-19 disease can manifest with intussusception in pediatric patients, but prevalence of abnormalities on ultrasounds performed for intussusception is uncertain. We aim to report our experience in children with COVID-19 presenting with suspected intussusception imaged with ultrasound.

Methods Children under 18 years who had an ultrasound for possible intussusception underwent retrospective analysis and were tested for COVID-19 between April 1 and December 14, 2020. Patients' demographic, clinical, radiological and surgical characteristics were reviewed.

Results Twenty-four COVID-19-positive patients were identified; 19 boys with mean age 3 years (range: 3 months-18 years). Ultrasound was abnormal in 11 patients $(11 / 24,46 \%)$. Sonographic features of enterocolitis were documented in seven children $(7 / 24,29 \%)$. Three boys $(3 / 24,13 \%)$ were found to have ileocolic intussusception on ultrasound and underwent air enema with failed reduction $(3 / 3,100 \%)$, precipitating surgical reductions, all with favorable outcomes. One patient $(1 / 24$, $4 \%$ ) was found to have a long segment of persistent small bowel-small bowel intussusception which was surgically repaired. Conclusion Given the known association between failed reduction at air enema and delayed presentation, heightened awareness for intussusception in the setting of COVID-19 should be maintained, though more often, the etiology was attributed to other GI manifestations of COVID-19.
\end{abstract}

Keywords Intussusception · COVID-19 $\cdot$ Children $\cdot$ Enema reduction

Rida Salman and Andrew C. Sher equally contributed to this manuscript.

Victor J. Seghers

vjsegher@texaschildrens.org

Rida Salman

rxsalman@texaschildrens.org

Andrew C. Sher

acsher@texaschildrens.org

Marla B. K. Sammer

mbsammer@texaschildrens.org

J. Ruben Rodriguez

rxrodri2@texaschildrens.org

Sohail R. Shah

srshah2@texaschildrens.org

1 Edward B. Singleton Department of Radiology, Texas Children's Hospital, 6701 Fannin St., Suite 470, Houston, TX 77030, USA

2 Department of Surgery, Baylor College of Medicine and Texas Children's Hospital, 6701 Fannin St., Suite 470, Houston, TX 77030, USA

\section{Introduction}

Gastrointestinal (GI) manifestations of severe acute respiratory syndrome coronavirus 2 (SARS-CoV-2) have been documented in nearly $25 \%$ of children with COVID-19 and ranged from mild symptoms including diarrhea, nausea, vomiting, and abdominal pain to more severe "surgical" presentations mimicking appendicitis [1, 2]. Intussusception has also been reported as a GI manifestation of COVID-19 [3-7].

Intussusception is the most common cause of acute bowel obstruction in young children, most commonly occurring between the ages of 5 and 10 months [8]. Several viral pathogens have been linked to intussusception as possible etiologies, namely adenovirus and human herpesvirus 6 (HHV-6) [9]. Five case reports from China, Pakistan, United Kingdom, Spain and the United States of America [3-7] suggest a plausible correlation between intussusception and SARSCoV-2 infection. 
Here, we report our experience with SARS-CoV2-positive children presenting with symptoms suspicious for intussusception who were imaged with ultrasound at a large pediatric tertiary care health system. The reduction rates for those with ileocolic intussusception were compared to historical institutional reduction rates, and correlated with duration of symptoms prior to presentation. In addition, we report the ultrasound findings of SARS-CoV2-negative children who were imaged with ultrasound for intussusception as well as the overall rate of GI symptoms in SARS-CoV-2-positive children admitted to our hospital during the study period.

\section{Methods}

\section{Data acquisition}

Patients were identified via Electronic Medical Record search, which included all patients who tested positive or negative for SARS-CoV-2 via antigen or antibody, as well as all their imaging exams. A retrospective chart review was performed for all children under 18 years who tested positive or negative for SARS-CoV-2 and underwent ultrasound (US) for possible intussusception between the local onset of the COVID-19 pandemic on April 1st, 2020 and December 14th, 2020. We recorded the demographic characteristics including age, gender and ethnicity, symptoms and duration of symptoms, ultrasound and when performed, enema imaging findings, fluoroscopy time of the enema reduction procedure, surgical reports, length of hospital stay and discharge summary on surgical patients.

Our institution uses a unique imaging order code to indicate that intussusception is suspected, which enables a dedicated "intussusception ultrasound" to be performed specifically for this indication. Other types of abdominopelvic ultrasounds such as abdominal ultrasound, right upper quadrant ultrasound, appendicitis ultrasound, or volvulus ultrasound, each with their unique protocols, were not included in this review as they are not utilized in patients suspected of having intussusception. Determination of SARS-CoV-2 positivity in our cohort was made by real-time reverse transcriptase (RT-PCR) analysis of nasopharyngeal swab samples. Patients who were imaged with US for intussusception but did not have a COVID-19 test, were excluded $(n=373)$.

We also examined the GI symptom rate among pediatric patients presenting with COVID-19, defined as the rate of patients who had ICD-10 diagnosis codes involving signs and symptoms of the digestive system and abdomen as part of their discharge summary reports.

\section{Intussusception ultrasound and air enema reduction}

The ultrasound protocol to look for intussusception consists of scanning bowel in the four abdominal quadrants using a high-frequency linear probe. If ileocolic intussusception is confirmed on ultrasound, the patient is scheduled for fluoroscopic enema reduction to be performed within $2 \mathrm{~h}$ of order placement. Fluoroscopic enema reduction is available 24/7 at all hospital locations and performed by a radiology-attending physician. Contraindications to fluoroscopic reduction are evaluated by the medical team and radiologist, including signs of peritonitis, shock and pneumoperitoneum. In our institution, the overwhelming majority of cases are performed via pneumatic (air) enema reduction although a small minority of faculty perform liquid enema reduction.

\section{Comparison to local historical data}

The failed fluoroscopic reduction rate in our institution has previously been reported at $13.9 \%$ and the time between fluoroscopic reduction attempt and surgical intervention ranged between 1.1 and $15.5 \mathrm{~h}$ (mean $4.3 \mathrm{~h}$ ) in hemodynamically stable children [10].

\section{Results}

During the study period, 685 patients were admitted with a positive COVID-19 test. 145 patients (21\%) were affected by GI symptoms. Of these, 24 SARS-CoV-2-positive patients who underwent intussusception ultrasound were identified ( 19 boys) with mean age 3 years (range: 3 months-18 years). All patients experienced one or more of the following symptoms: abdominal pain, vomiting, diarrhea (bloody or not), intermittent crying, and decreased oral intake.

Abnormal ultrasound was noted in 11 patients (11/24, $46 \%)$. Sonographic features of enteritis or enterocolitis (Fig. 1) were documented sonographically in seven children $(7 / 24,29 \%)$. One patient $(1 / 24,4 \%)$, a 3 -year-old boy, was found to have a long segment $(4.7 \mathrm{~cm})$ of small bowel-small bowel intussusception in the left upper abdominal quadrant which was still present on a repeat ultrasound $4 \mathrm{~h}$ later and underwent surgical reduction which showed an internal hernia defect through which a large amount of small bowel had herniated without vascular compromise (Fig. 2). The bowel was successfully reduced and placed back in its orthotopic position. Table 1 outlines the demographic and clinical characteristics of these eight children.

Three boys $(3 / 24,13 \%)$ were diagnosed with ileocolic intussusception (Fig. 3) via ultrasound and all experienced 

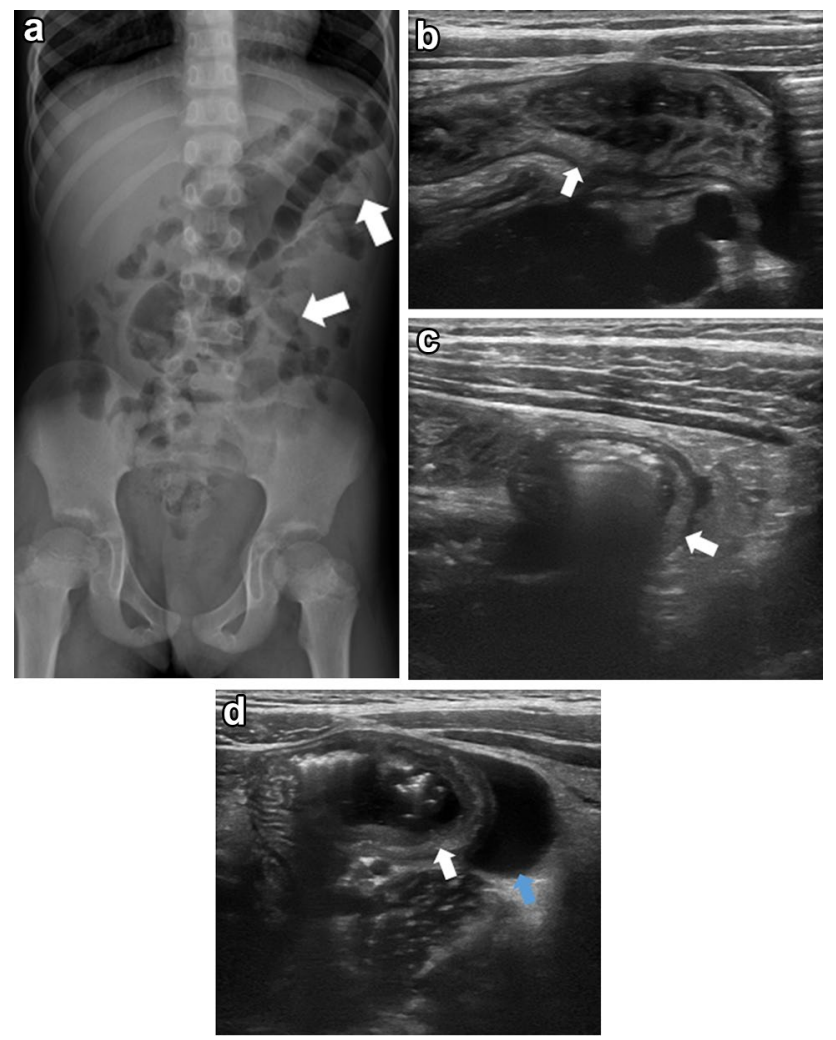

Fig. 1 Seven-year-old Asian female presented with 1-day history of abdominal pain, vomiting and bloody stool. Frontal abdominal view (a) shows thickened bowel wall (arrows). Transverse ultrasound images (b-d) show bowel wall thickening (arrow) with associated simple free fluid (blue arrow). Findings are consistent with enterocolitis failed air enema reduction with respective fluoroscopy times of 16, 17 and 21 min (mean $18 \mathrm{~min}$ ). Two patients are Hispanic (67\%) and one is white (33\%) with their symptoms' duration ranged between 24 and $96 \mathrm{~h}$ (mean $56 \mathrm{~h}$ ). One patient underwent a repeat fluoroscopic enema reduction attempt $8 \mathrm{~h}$ later with partial reduction to the level of ileocecal valve. These patients all subsequently underwent successful surgical reduction in the operating room without complications with a time range of 1.8-13 h (mean $5.5 \mathrm{~h}$ ) between the first fluoroscopic reduction attempt and surgical reduction compared to an institutional range of 1.1-15.5 h (mean $4.3 \mathrm{~h}$ ) [10]. Two patients had surgery $1.8 \mathrm{~h}$ after the failed reduction enema and one child had surgery $13 \mathrm{~h}$ after the first attempt and $5 \mathrm{~h}$ after the second attempt; this child remained clinically stable without distress. None of these patients had evidence of bowel ischemia nor required bowel resection and all were discharged home on postoperative day 2 in stable condition. Table 2 summarizes the demographic and clinical characteristics of these three children.

Four hundred and three SARS-CoV-2-negative patients who underwent intussusception ultrasound were identified (248 boys) with mean age 3 years (range: 1 month-17 years). Abnormal ultrasound findings were noted in 117 patients (117/403, 29\%), including enteritis or enterocolitis (58/403, $14 \%)$, ileocolic intussusception $(18 / 403,4 \%)$, transient small bowel-small bowel intussusception $(18 / 403,4 \%)$, gallbladder sludge/gallstone $(7 / 403,1.7 \%)$, appendicitis $(5 / 403$, $1.2 \%)$, small bowel obstruction $(3 / 403,0.7 \%)$, complex ascites $(2 / 403,0.5 \%)$, volvulus $(1 / 403,0.2 \%)$, hypertrophic pyloric stenosis $(1 / 403,0.2 \%)$, pancreatitis $(1 / 403,0.2 \%)$,
Fig. 2 Three-year-old white male presented with 4-day history of vomiting but no fever or cough. Greyscale sagittal and transverse ultrasound images $(\mathbf{a}, \mathbf{b})$ reveal a long segment $(4.7 \mathrm{~cm})$ of small bowel intussusception in the left upper quadrant (arrow). Flow is documented within the intussusceptum (arrow) on the color Doppler sagittal ultrasound image (c). An image taken during surgical intervention (d) shows the hernia defect (arrow) through which the small bowel loops herniated
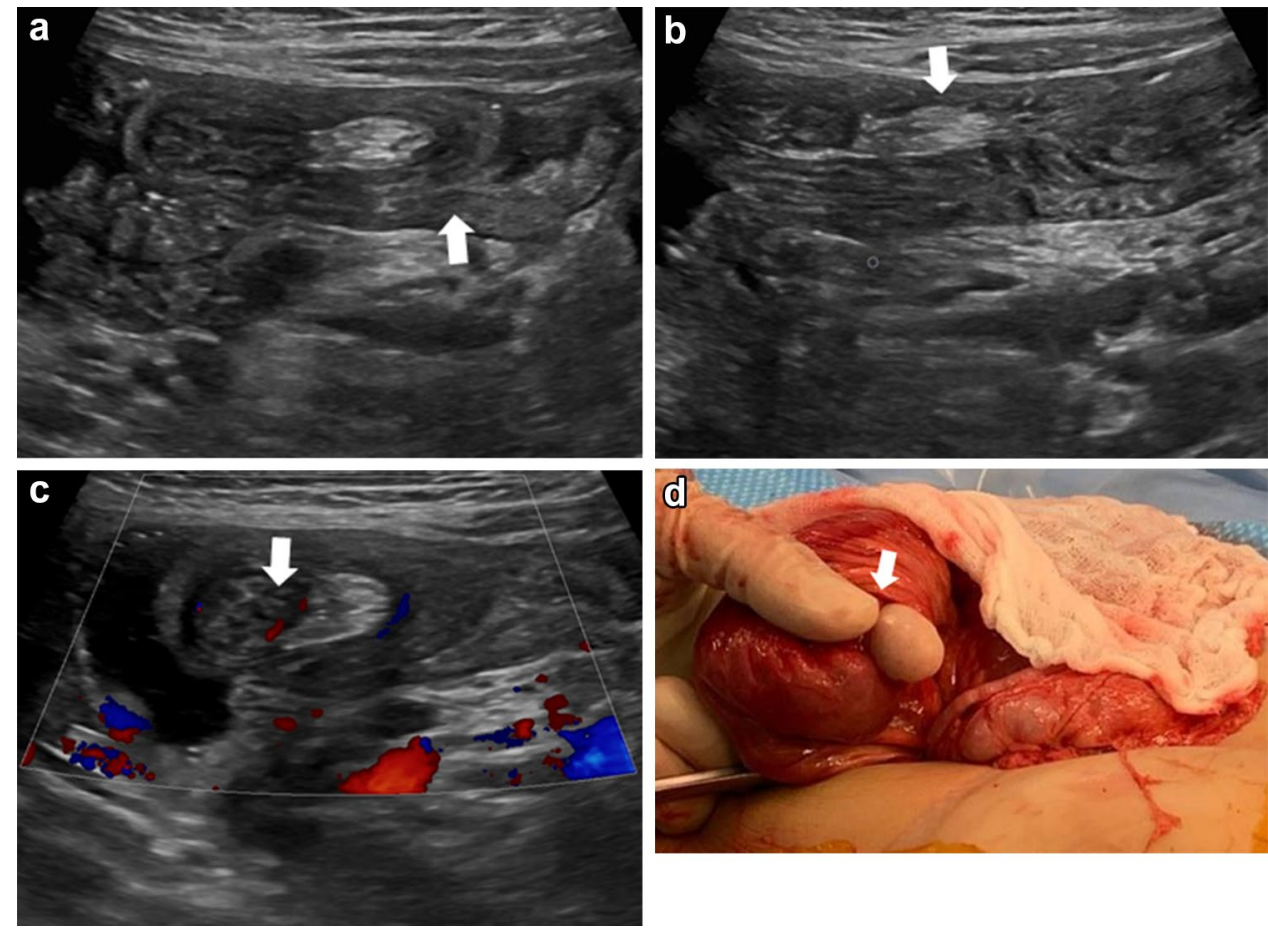
Table 1 Demographic and clinical characteristics of children with COVID-19 and enterocolitis and small bowel intussusception (M: male, F: female)

\begin{tabular}{|c|c|c|c|c|c|c|}
\hline & Age & Sex & Ethnicity & Gastrointestinal symptoms, duration & $\begin{array}{l}\text { Respiratory symp- } \\
\text { toms, duration }\end{array}$ & Fever, duration \\
\hline 1 & 3 years & M & White & Vomiting, $96 \mathrm{~h}$ & None & No \\
\hline 2 & 7 years & $\mathrm{F}$ & Asian & Abdominal pain, vomiting and bloody stool, $24 \mathrm{~h}$ & None & No \\
\hline 3 & 3 years & M & White & Abdominal pain, vomiting and bloody stool, $24 \mathrm{~h}$ & None & Yes, $48 \mathrm{~h}$ \\
\hline 4 & 5 years & M & Hispanic & Vomiting and diarrhea, $72 \mathrm{~h}$ & None & Yes, $72 \mathrm{~h}$ \\
\hline 5 & 1 year & M & Hispanic & Vomiting, $96 \mathrm{~h}$ & None & No \\
\hline 6 & 8 years & M & White & Abdominal pain and bloody diarrhea, $48 \mathrm{~h}$ & None & No \\
\hline 7 & 4 months & M & Hispanic & Vomiting, $168 \mathrm{~h}$ & None & Yes, $48 \mathrm{~h}$ \\
\hline 8 & 5 months & M & Hispanic & Bloody diarrhea, $24 \mathrm{~h}$ & None & No \\
\hline
\end{tabular}
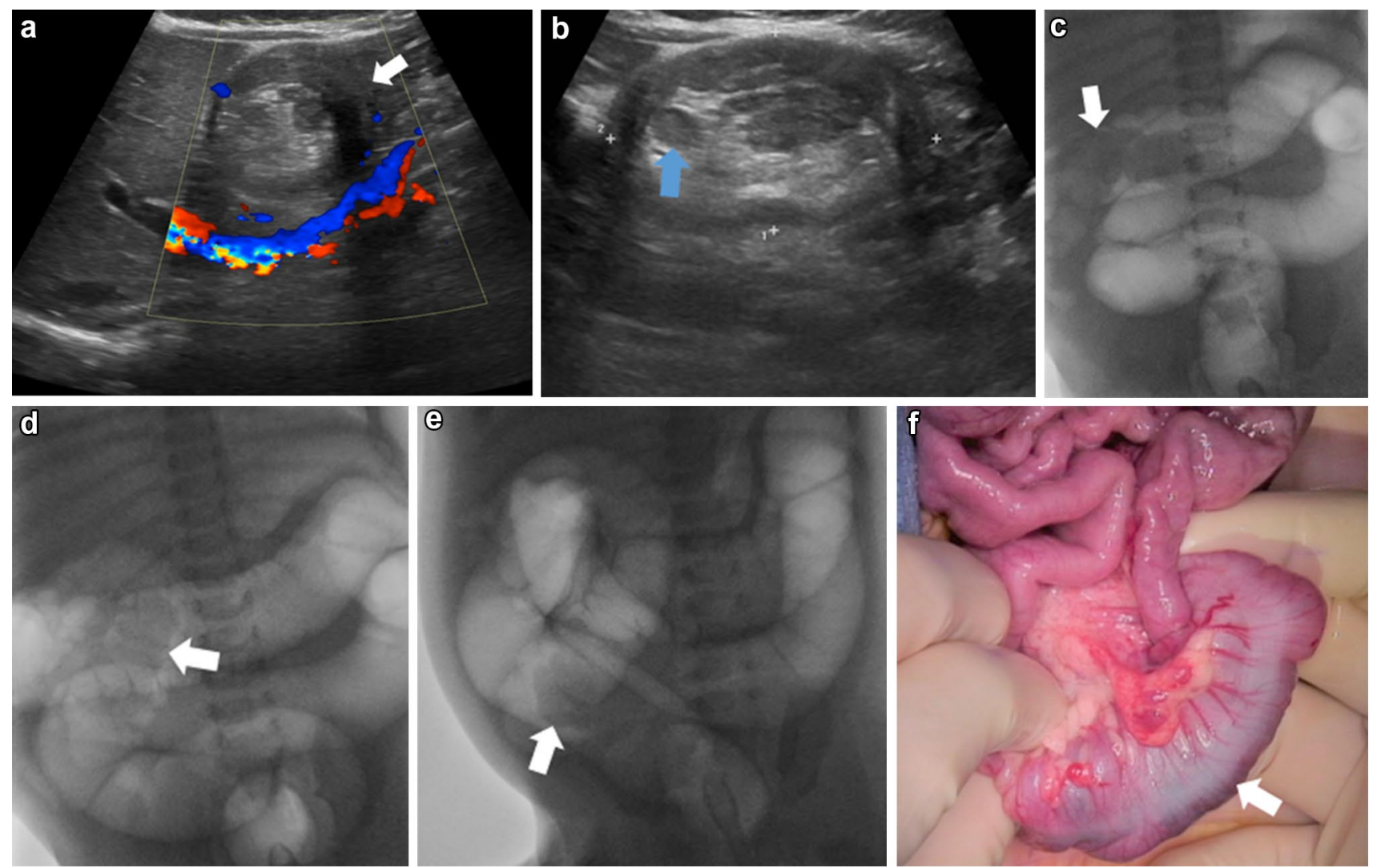

Fig. 3 3-month-old white male presented with 4-day history of bloody diarrhea but no fever or cough. Color Doppler transverse and greyscale sagittal ultrasound images (a and b) show a bowel within bowel appearance in the right upper quadrant (arrow) compatible with ileocolic intussusception containing multiple lymph nodes and mesenteric fat (blue arrow). Frontal fluoroscopic views from the air

splenic infarct $(1 / 403,0.2 \%)$, hydronephrosis $(1 / 403,0.2 \%)$ and infected urachal cyst $(1 / 403,0.2 \%)$.

Among the 18 SARS-CoV-2-negative patients diagnosed with ileocolic intussusception (14 boys), 9 are Hispanic (50\%), 6 are white (33\%) and 3 are black (17\%). enema (c-e) show progressive but incomplete reduction of the intussusception to the level of ileocecal valve (arrow). An image taken during surgical procedure (f) shows ecchymotic and edematous but completely viable bowel (arrow). There was a standard ileocolic intussusception which was manually reduced. No enterectomy was performed

Their symptoms' duration ranged between 1 and $120 \mathrm{~h}$ (mean 35 h). 15 cases (83\%) had successful air enema reduction while 3 patients (17\%) had partial fluoroscopic reduction attempts and underwent successful surgical reduction. Bowel ischemia was found in one boy who 
Table 2 Demographic and clinical characteristics of children with COVID-19 and ileocolic intussusception (M: male)

\begin{tabular}{|c|c|c|c|c|c|c|c|c|}
\hline & Age & Sex & Ethnicity & Gastrointestinal symptoms, duration & $\begin{array}{l}\text { Respiratory } \\
\text { symptoms, } \\
\text { duration }\end{array}$ & Fever, duration & COVID-19 diagnosis & $\begin{array}{l}\text { Ultrasound, } \\
\text { fluoroscopy, } \\
\text { surgery }\end{array}$ \\
\hline 1 & 3 months & M & White & Bloody diarrhea, $96 \mathrm{~h}$ & None & No & $9 / 30 / 2020$ & $9 / 30 / 2020$ \\
\hline 2 & 1 year & M & Hispanic & $\begin{array}{l}\text { Abdominal pain and bloody diar- } \\
\text { rhea, } 24 \mathrm{~h}\end{array}$ & Cough, $48 \mathrm{~h}$ & Yes, $48 \mathrm{~h}$ & $7 / 5 / 2020$ & $7 / 8 / 2020$ \\
\hline 3 & 4 years & M & Hispanic & Abdominal pain and vomiting, $48 \mathrm{~h}$ & Cough, $48 \mathrm{~h}$ & Yes, $48 \mathrm{~h}$ & $6 / 25 / 2020$ & $6 / 27 / 2020$ \\
\hline
\end{tabular}

needed distal small bowel and right colon resection with ileocolic anastomosis.

\section{Discussion}

Nearly half of all intussusception ultrasounds performed for suspected intussusception in SARS-CoV-2-positive patients showed GI abnormalities on ultrasound, most commonly enterocolitis, and less commonly intussusception. In the three patients with ileocolic intussusception, we experienced a $100 \%$ failure rate of pneumatic reduction. Furthermore, the one patient with small bowel-small bowel intussusception also required surgical reduction.

Delay in diagnosis secondary to delayed presentation will lead to delay in providing the adequate non-invasive treatment through enema reduction and increase risk of failure. Increased risk of requiring surgical reduction and bowel resection has previously been reported with symptom duration exceeding 24-48 h [11, 12]. All three patients with failed reduction in our cohort presented to the emergency department 24 to $96 \mathrm{~h}$ (mean $56 \mathrm{~h}$ ) after experiencing GI symptoms, which may account for our failure rate.

Of note, our observation of failed enema reductions is not similar to what has been previously reported. Specifically, most COVID-19-associated intussusception cases have been successfully treated with non-surgical reduction $[3,4,6,7]$. There was only one reported case of failed pneumatic enema reduction requiring surgical intervention which also identified malrotation and was treated with both intussusception reduction and a Ladd's procedure [5]. The symptom duration in these patients ranged between 30 and $96 \mathrm{~h}$ (mean $55.5 \mathrm{~h}$ ) which is similar to the range and mean symptom duration of our patients, suggesting that delayed presentation is not the only factor accounting for our failed reduction rate. We do not think it likely that our failed reduction rate was related to radiologist concern over patient COVID-19-positive status since the enema reduction fluoroscopic time ranged between 16 and 21 min (mean $18 \mathrm{~min}$ ) with multiple attempts, which is much higher than established ranges for enema reduction, reported previously in the literature at $4.9 \mathrm{~min}$ for failed procedures [13].
One patient with small bowel intussusception also required surgical reduction which is atypical since most small bowel intussusceptions spontaneously reduce. However, surgical intervention is needed when there is an associated bowel necrosis or lead point, and when there is an intussusception length greater than $3.5 \mathrm{~cm}[14,15]$. In our case, an internal hernia defect was found to be the lead point for a long segment $(4.7 \mathrm{~cm})$ of small bowel intussusception.

One of the three children with ileocolic intussusception presented exclusively with GI symptoms, unlike the other two cases who initially experienced fever and cough before developing GI symptoms. This emphasizes the need to raise suspicion of SARS-CoV-2 infection in children with only GI manifestations as previously documented to avoid delayed diagnosis, increased risk of complications and more severe disease course [16].

Our finding of other GI manifestations on ultrasound performed for intussusception is not surprising given the potential selection bias of exams performed in children presenting with GI symptoms concerning for intussusception. However, the rate of abnormalities on ultrasound is still worth noting. In addition, 21\% of SARS-CoV-2-positive patients admitted to our hospital were affected by GI manifestations including intussusception. Published meta-analysis of studies examining gastrointestinal manifestations of COVID-19 in children documented prevalence ranging from 0 to $88 \%$ with a pooled prevalence of $22.8 \%$ [1]. In another pooled analysis, GI manifestations were reported in 12\% of children with COVID-19 [17]. Our concordant results support the growing recognition that GI manifestations of pediatric COVID-19 are common.

Several etiologies have previously been reported to be associated with intussusception including viral infections such as adenovirus. The pathophysiology involves the formation of lymphoid hyperplasia of Peyer's patches in the ileum as well as the presence of inflammatory neuropathy secondary to intestinal nervous plexus lymphocytic infiltration leading to abnormal bowel peristalsis which in turns causes bowel telescoping into each other [18]. Although it is not documented, the pathogenesis of SARS-CoV-2-related intussusception may also be similar to what is seen with adenovirus. Furthermore, the intestinal epithelial cells express the angiotensin-converting enzyme 2 (ACE-2) receptors and the transmembrane protease serine 2 (TMPRSS2) enzyme 
which mediate the entry of SARS-CoV-2. Enterocyte neuronal cells also express ACE-2 receptors $[19,20]$ which might also explain COVID-19 GI infection in our cohort, since almost $30 \%$ of children had sonographic evidence of enterocolitis. This is beyond the scope of this report, but suggests further area for study, including pathologic analysis of the bowel in cases where bowel may be compromised and resection performed.

Our study is subject to many limitations including its retrospective nature and being conducted in a single institution over a short period of time. Specifically, the cohort of SARSCoV-2-positive cases is small, and therefore, the ability to draw statistical inference from the available demographic and clinical data of the three children with intussusception is limited. In addition, there is large difference in patient numbers between SARS-CoV-2-positive $(n=24)$ versus SARS-CoV-2-negative patients $(n=403)$ who were imaged for intussusception limiting the ability to run an accurate statistical comparison between these two groups. While our group noted more variable diagnoses on ultrasound imaging of SARS-CoV-2-negative patients versus SARSCoV-2-positive patients, this can be explained by the larger number of imaged SARS-CoV-2-negative patients. While the failure rate of enema reduction in patients who tested negative for COVID-19 and had intussusception during the same period of time (17\%) is close to the known failure rate in our institution (13.9\%) [10], it is vastly different from the failure rate in SARS-CoV-2-positive patients (100\%). This significant difference in patients' outcome after fluoroscopic enema needs further evaluation with a larger cohort of SARS-CoV-2-positive patients with intussusception to assess for possible demographic and clinical factors playing a role in failure of enema reduction. Furthermore, our institution was not performing strain analysis during the study period to check for any possible propensity of a particular variant for GI involvement in children. Multi-institutional longitudinal studies are needed to elucidate the association between SARS-CoV-2 and GI disease, not limited to intussusception, in the pediatric population.

\section{Conclusion}

A substantial proportion of ultrasounds performed for intussusception in children with COVID-19 show abnormalities of the GI tract, further indicating that the gastrointestinal tract is not infrequently involved in children with COVID19. $13 \%$ of our patients $(3 / 24)$ were diagnosed with ileocolic intussusception, of which $100 \%$ failed enema reduction. Our findings emphasize the need for increased awareness about the possibility of GI symptoms to precede the respiratory symptoms of COVID-19 and also to be the only disease manifestation in children. Given the known increased failure rate with delayed diagnosis of intussusception, heightened suspicion for ileocolic intussusception should be maintained in children diagnosed with COVID-19.

Acknowledgements The Texas Children's Hospital COVID-19 Imaging Taskforce (Ananth V. Annapragada, Nilesh K. Desai, R. Paul Guillerman, Thierry A.G.M. Huisman, Prakash M. Masand, Rida Salman, Gunes Orman, Amir H. Pezekhmehr, Marla B. Sammer, and Victor J. Seghers).

Author contributions All the authors whose names appear on the submission. RS made substantial contributions to the conception or design of the work, including acquisition, analysis, or interpretation of data. ACS drafted the work or revised it critically for important intellectual content; MBKS approved the version to be published; and JRR agree to be accountable for all aspects of the work in ensuring that questions related to the accuracy or integrity of any part of the work are appropriately investigated and resolved.

Funding No funds, grants, or other support was received.

\section{Declarations}

Conflict of interest The authors have no relevant financial or non-financial interests to disclose.

Ethical approval Our institutional review board approved this retrospective study, and patient consent was waived.

\section{References}

1. Akobeng AK, Grafton-Clarke C, Abdelgadir I, Twum- Barimah E, Gordon M (2020) Gastrointestinal manifestations of COVID19 in children: a systematic review and meta-analysis. Frontline Gastroenterol. https://doi.org/10.1136/flgastro-2020-101529

2. Bolia R, Ranjan R, Bhat NK (2021) Recognizing the gastrointestinal manifestation of pediatric coronavirus disease 2019. Indian J Pediatr 88(1):101-102. https://doi.org/10.1007/ s12098-020-03481-y

3. Cai X, Ma Y, Li S, Chen Y, Rong Z, Li W (2020) Clinical characteristics of 5 COVID-19 cases with non-respiratory symptoms as the first manifestation in children. Front Pediatr 8:258. https:// doi.org/10.3389/fped.2020.00258

4. Moazzam Z, Salim A, Ashraf A, Jehan F, Arshad M (2020) Intussusception in an infant as a manifestation of COVID-19. J Pediatr Surg Case Rep 59:101533. https://doi.org/10.1016/j.epsc.2020. 101533

5. Makrinioti H, MacDonald A, Lu X et al (2020) Intussusception in 2 children with severe acute respiratory syndrome coronavirus-2 infection. J Pediatric Infect Dis Soc 9(4):504-506. https://doi.org/ 10.1093/jpids/piaa096

6. Martínez-Castaño I, Calabuig-Barbero E, Gonzálvez-Piñera J, López-Ayala JM (2020) COVID-19 infection is a diagnostic challenge in infants with ileocecal intussusception. Pediatr Emerg Care 36(6):e368. https://doi.org/10.1097/PEC.0000000000002155

7. Bazuaye-Ekwuyasi EA, Camacho AC, Saenz Rios F et al (2020) Intussusception in a child with COVID-19 in the USA. Emerg Radiol 27(6):761-764. https://doi.org/10.1007/ s10140-020-01860-8

8. Ko HS, Schenk JP, Tröger J, Rohrschneider WK (2007) Current radiological management of intussusception in 
children. Eur Radiol 17(9):2411-2421. https://doi.org/10.1007/ s00330-007-0589-y

9. Burnett E, Kabir F, Van Trang N et al (2020) Infectious etiologies of intussusception among children $<2$ years old in 4 Asian countries. J Infect Dis 221(9):1499-1505. https://doi.org/10.1093/ infdis/jiz621

10. Nguyen HN, Kan JH, Guillerman RP, Cassady CI (2014) Intussusception revisited: is immediate on-site surgeon availability at the time of reduction necessary? Am J Roentgenol 202(2):432-436. https://doi.org/10.2214/AJR.13.10731

11. Kaiser AD, Applegate KE, Ladd AP (2007) Current success in the treatment of intussusception in children. Surgery 142(4):469-477. https://doi.org/10.1016/j.surg.2007.07.015

12. Fallon SC, Lopez ME, Zhang W et al (2013) Risk factors for surgery in pediatric intussusception in the era of pneumatic reduction. J Pediatr Surg 48(5):1032-1036. https://doi.org/10.1016/j. jpedsurg.2013.02.021

13. Lui KW, Wong HF, Cheung YC et al (2001) Air enema for diagnosis and reduction of intussusception in children: clinical experience and fluoroscopy time correlation. J Pediatr Surg 36(3):479_ 481. https://doi.org/10.1053/jpsu.2001.21604

14. Munden MM, Bruzzi JF, Coley BD, Munden RF (2007) Sonography of pediatric small-bowel intussusception: differentiating surgical from nonsurgical cases. Am J Roentgenol 188(1):275-279. https://doi.org/10.2214/AJR.05.2049

15. Chang TH, Wu JL, Chang LY (2020) Clinical characteristics and diagnostic challenges of pediatric COVID-19: a systematic review and meta-analysis. J Formos Med Assoc 119(5):982-989. https:// doi.org/10.1016/j.jfma.2020.04.007
16. Mao R, Qiu Y, He JS et al (2020) Manifestations and prognosis of gastrointestinal and liver involvement in patients with COVID19: a systematic review and meta-analysis. Lancet Gastroenterol Hepatol 5(7):667-678. https://doi.org/10.1016/S2468-1253(20) 30126-6

17. Lioubashevsky N, Hiller N, Rozovsky K, Segev L, Simanovsky N (2013) Ileocolic versus small-bowel intussusception in children: can US enable reliable differentiation? Radiology 269(1):266271. https://doi.org/10.1148/radiol.13122639

18. Kaemmerer E, Tischendorf JJ, Steinau G, Wagner N, Gassler N (2009) Ileocecal intussusception with histomorphological features of inflammatory neuropathy in adenovirus infection. Gastroenterol Res Pract 2009:579501. https://doi.org/10.1155/2009/579501

19. Villapol S (2020) Gastrointestinal symptoms associated with COVID-19: impact on the gut microbiome. Transl Res 226:57-69. https://doi.org/10.1016/j.trs1.2020.08.004

20. Lee JJ, Kopetz S, Vilar E, Shen JP, Chen K, Maitra A (2020) Relative abundance of SARS-CoV-2 entry genes in the enterocytes of the lower gastrointestinal tract. Genes 11(6):645. https://doi.org/ $10.3390 /$ genes 11060645

Publisher's Note Springer Nature remains neutral with regard to jurisdictional claims in published maps and institutional affiliations. 